\title{
Simulador de Reconhecedores de Palavras Isoladas
}

\author{
ALI HUSSEIN SAYED \\ NORMONDS ALENS
} Neste trabalho é apresentado um simulador de reconhecedores de comandos da fala [1].
O similador permite testar vários algoritmos propostos na literatura e variaços destes
algoritmos.

\section{INTRODUÇÃO}

A área de reconhecimento de voz preocupa-se em prover meios de comunicaçāo com as máquinas através da fala humana. Estudos recentes mostram que a comunicaçāo através da fala é o meio preferido pelas pessoas e que tenderá a ser uma alternativa barata para a entrada de dados em computadores $[2,3]$. O simulador desenvolvido neste trabalho abrange a área de reconhecimento de comandos da fala. O sistema opera com vocabulário limitado e deve ser previamente treinado. Testes realizados com o auxilio do simulador desenvolvido, para uma configuraçāo freqüente na literatura, (ver seçáo 19), forneceram taxas de acerto entre $95 \%$ e $98 \%$.

\section{APARELHO FONADOR HUMANO}

O primeiro passo no processamento do sinal de voz consiste em modelar o aparelho fonador humano. $O$ aparelho fonador constitui-se de um tubo acústico que se estende entre a glote e os lábios. A cavidade nasal pode ser acoplada ou náo à cavidade oral através da movimentação da úvula. O tubo acústico possui características e dimensōes variáveis em funçāo da pessoa e do som emitido [11]. Os sons classificam-se em sons vocálicos (ex. vogais), sons oclusivos $(\mathrm{pl} /, / \mathrm{t})$, sons fricativos $(\mathrm{Vs} /, / f)$ e sons nasais $(\mathrm{V} /, / \mathrm{m} /)[11,12]$. 
A figura 1 mostra o sinal acústico do dígito "oito", onde pode-se observar as regiōes vocálicas e as regiōes de "silêncio" da palavra. O termo "silêncio" refere-se ao ruído de fundo do ambiente acústico do reconhecedor.

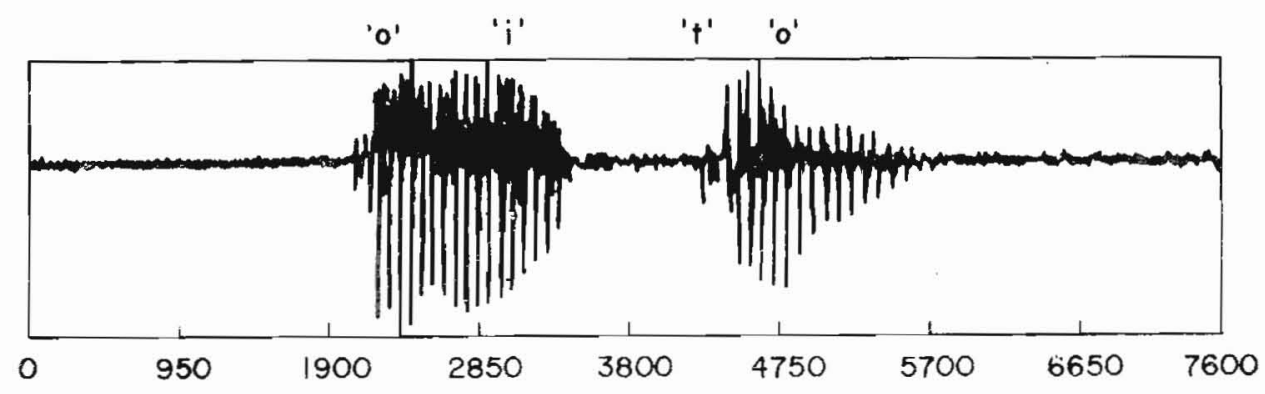

Figura 1. Sinal acústico do dígito "oito". Sāo 7600 amostras relativas a uma taxa de amostragem de $8 \mathrm{KHz}$.

Resuiltados experimentais mostram que as características co sistema de geraçăo do sinal de voz mudam lentamente. A mudança ocorre em períodos em torno de 10 a $30 \mathrm{~ms}[8,12,13,14,15]$. A Figura 2 mostra um intervalo do som "oi" do dígito "oito". Percebe-se que o sir:al nāo apresenta mudanças bruscas de comportamento. Pode-se, entāo, modelar o aparelho fonador humano por um sistema iinear e lentamente variável com o tempo [12, 13], que pode ser excitado tanto por um trem de impulsos quasi-periódicos (no caso de sons vocálicos) ou por ruído branco (no caso de sons nāo-vocálicos), conforme Figura 3. Filtrando o sinal de voz proveniente do microfone com um filtro $L(z)$ do tipo:

$$
L(z)=1-\mu z^{-1}
$$

obtém-se um sistema global V(z), com os efeitos da radiaçāo nos lábios e da variação da área da glote reduzidos. A este processo de tratamento do sinal de voz, dá-se o nome de pré-ênfase, conforme Figura 4. O parâmetro $\mu$ é denominado fator de pré-ênfase e o seu valor pode ser programado pelo SIR PI (SImulador de Reconhecedores de Palavras Isoladas) (valores típicos de $\mu$ são próximos de 1,0 ). 


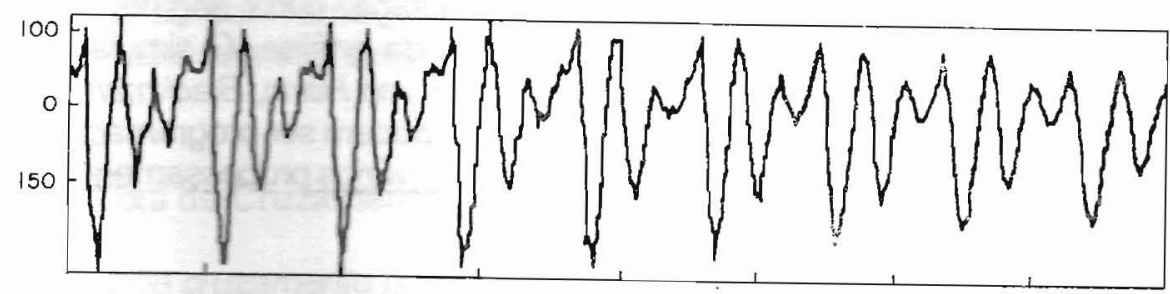

Figura 2. Intervalo do som "oi" do dígito "oito"

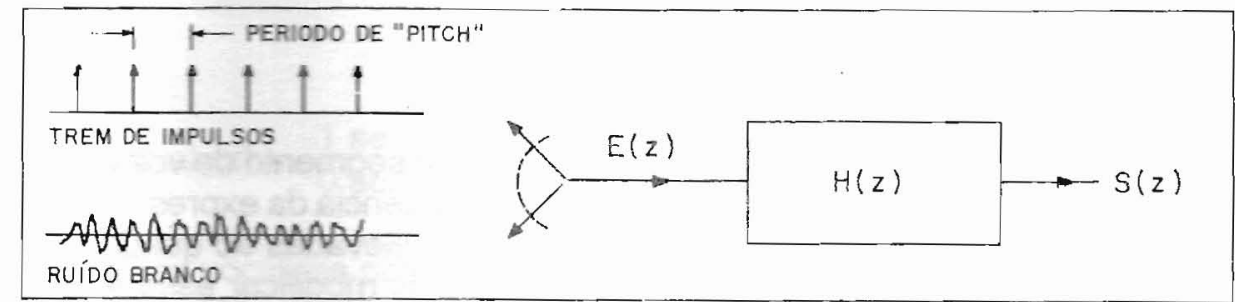

Figura 3. Modelo linear e lentamente variável (variável quadro a quadro).

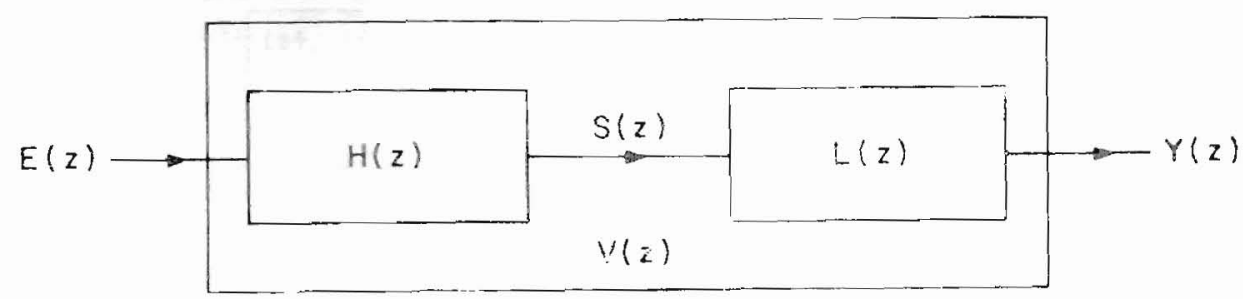

Figura 4. Modelo com pré-ênfase

Tal modelo, adotado neste trabalho, tem-se mostrado eficiente e funciona bem na prática [8].

Pavista da Sociodade Brasileira de Telecomunicaçōes 


\section{PROCESSAMENTO TEMPORAL DO SINAL DE VOZ}

O sinal de voz pode ser analisado em intervalos de duraçáo curta, segmentando-o utilizando janelas de duração igual à duraçāo da análise. $O$ simulador permite escolher entre as janelas do tipo Retangular, von Hann, Blackmann e Bartlett [16]. Alagura $\mathrm{N}$ e o deslocamento $\mathrm{D}$ da janela podem ser programados. Após o janelamento, o segmento de voz é submetido a vários processamentos:

\subsection{Medida de Energia de Tempo Curto}

A amplitude dos sons nāo-vocálicos é bem menor que a amplitude dos sons vocálicos. A "energia" quadrática de tempo curto, $E(n)$, fornece uma boa representaçāo desta variação de amplitude [12, 15]:

$$
E(n)=\sum_{m=0}^{N-1}|y(n-m) w(m)|^{2}
$$

onde w(m) é uma janela de largura $N$ e y $(n-m)$ é um segmento de voz entre $n+N+1$ e n para $0 \leq m \leq N-1$. A principal inconveniência da expressão (2) é o destaque dado às grandes amplitudes (que são elevadas ao quadrado) em relação aos níveis mais baixos. Uma maneira de modificar esse efeito seria utilizar a medida de "energia" absoluta de tempo curto, $M(n)$ :
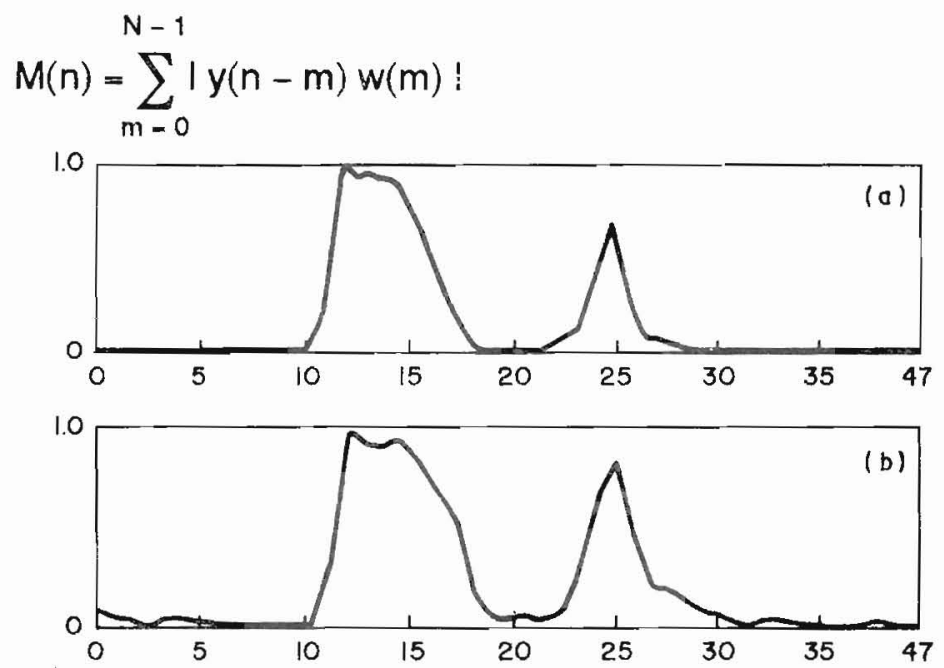

Figura 5. "Energias" quadrática (a) e absoluta (b) do dígito "oito" em função de segmentos de $20 \mathrm{~ms}$ ( $N=160$ amostras). 
O simulador permite escolher entre a medida absoluta e a medida quadrática. A Figura 5 mostra as "energias" absoluta e quadrática do dígito "oito", calculadas com uma janela retangular de largura $N=160$ amostras $e$ deslocada sem sobreposições $(D=160)$.

\subsection{Taxa de Cruzamento por Zero de Tempo Curto}

A taxa de cruzamento por zero, $z(n)$, ao loingo do sinal de voz, fornece uma idéia robusta do conteúdo espectral do sinal $[12,15,17]$. $Z(n)$ é definida por:

$$
Z(n)=\sum_{m=0}^{N-1}|\operatorname{sina}|\{y(n-m)\}-\sin a|\{y(n-m-1)\}| w(m)
$$

onde

$$
\text { Sinal }(x)=\begin{aligned}
& 1 \text { se } x \geq 0 \\
& 0 \text { se } x<0
\end{aligned}
$$

As regiōes vocálicas possuem taxas de cruzamento por zero menores que as regiōes não vocálicas. A Figura 6 mostra a taxa de cruzamento de zero do dígito "sito", calculada com uma janela retangular de largura $N=160$ amostras e deslocada sem sobreposiçōes.

Deve-se mencionar que o nível $\mathrm{DC}$ e interferências de $60 \mathrm{~Hz}$ prejudicam: o cálculo de Z.(n) [12]. O simulador SIR PI permite controlar o nível DC do sinal de voz.

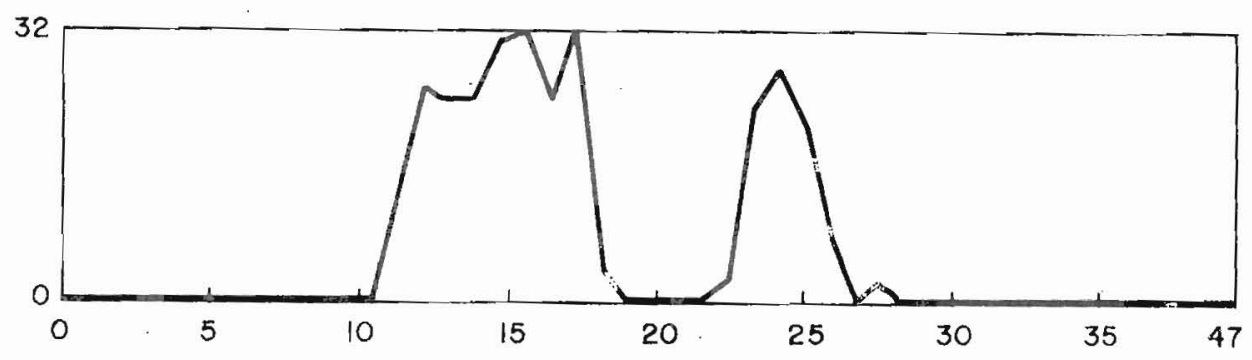

Figura 6. Taxa de cruzamento de zero do dígito "oito" em h̆:nção de segmentos de $20 \mathrm{~ms}$ ( $N=160$ amostras) 


\subsection{Função de Autocorrelação de Tempo Curto}

A função de autoccirelação de tempo curto, $R(k)$, é utilizada na análise do sinal de voz pela técnica de predição linear. O coeficiente de autocorrelação de índice $k$ é dado por [12].

$$
\begin{aligned}
& v_{n}(m)=y(n-m) w(m), \quad 0 \leq m \leq \mathbb{N}-1 \\
& R(k)=\sum_{m=0}^{N-1-k} v_{n}(m) v_{n}(m+k)
\end{aligned}
$$

onde $v_{n}(m)$ é um segmento janelado do sinal de voz, de lagura $N$. As amostras que compóem $v_{n}(m)$ vão de $y(n-\hat{N}+1)$ a $y(n)$.

\section{PONTOS EXTREMOS DA PALAVRA}

A localização dos pontos extremos do comando pronunciado, é um fator importante que permite reduzir o número de amostras a serem analisadas pelo sistema e que afeta consideravelmente a sua taxa de acerto de reconhecimento. $O$ alggritmo empregado foi sugerido por Rabiner e Sambur [18] e caracteriza-se pela sua simplicidade. $O$ algoritmo emprega dois parâmetros: a "energia" de tempo curto, $E(n)$ ou $M(n)$, e a taxa de cruzamento por zero, $Z(n)$, do sinal de voz. Estes parâmetros são utilizados na determinaçāo de limiares que controlam o processo de decisão. São definidos dois limiares de energia $L_{\text {es }}$ e $L_{0 i}$ (superior e inferior respectivamente) e um limiar de cruzamento de zero $\mathrm{L}_{z c}$. Uma vez determinados os limiares, o algoritmo parte para a localizaçāo dos extremos. O algoritmo realiza a busca a partir do início da palavra segundo o critério de energia. O segmento $\mathrm{N}$ 1, em que a energia excede o limiar inferior $L_{e i}$ e continua subindo até exceder $L_{e s}$, éadotado como estimativa inicial para o começo da palavra. O mesmo procedimento é repetido para a licalizaçāo da estimativa N2 do final da palavra. As estimativas N1 e N2 são então corrigidas de acordo com o critério da taxa de cruzamento de zero. Neste caso, parte-se de $\mathrm{N} 1$ em direção ao início da palavra e determina-se o número de vezes em que $Z(n)$ ultrapassa $L_{z c r}$ ema segmentos anteriores a N1. Se este número exceder 3 , a estimativa inicial N1 é deslocada até o primeiro ponto, no tempo, em que $Z(n)$ ultrapassa $L_{z \alpha}$. O mesmo procedimento é repetido para $\beta$ segmentos posteriores à estimativa N2. O número de segmentos $\alpha$ e $\beta$ devem ser escolhidos adequadamente (valores típicos são $\alpha=\beta=25$ ). O simulador SIR PI permite controlar o cálculo dos 
limiares e portanto, permite controlar a precisáo do algoritmo. Aplicando o algoritmo ao dígito "oito" (janela retangular com $N=160$ e deslocamento sem sobreposiçäo), resultam os pontos extremos $(N 1=10$ e $N 2=30)$ indicados na figura 7.

"ENERGIA" (NORMALIZADA)

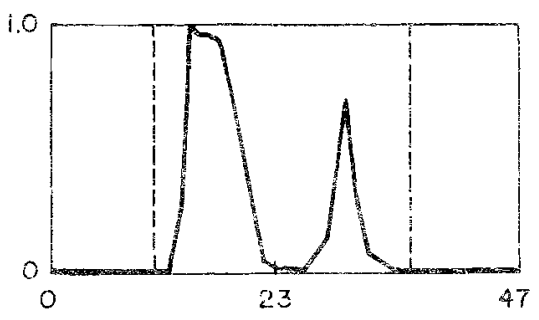

TAXA DE CRUZAMENTO POF ZERO

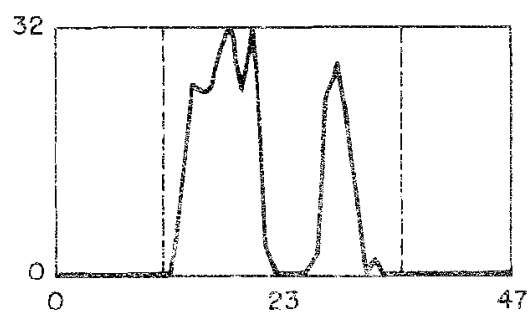

$N=10 \Leftrightarrow N 2=30$

Figura 7. Pontos extremos do digito "oito"

\section{PREDIÇÄO UINEAR}

A análise da voz por prediçäo linear, baseia-se na hipótese de que uma amostra do sinal de voz pode ser aproximada por uma combinaçäo linear das amostras anteriores ou das amostras futuras [14]. Uma preciçäo de ordem $P$, procura estimar amosira $y_{w}(n)$, do instante $n$, 2 partir de $P$ amostras anionors. Ondice winclca janelamento da amostra y (n). Pode-se, também. estmar a amostrs $y_{w}(n-P)$, do instante $p$ - a partir de $p$ amostras futuras:

$$
\begin{aligned}
& \bar{y}(n)=\sum_{k=1}^{F} \bar{a}_{k} y_{y}(n-k) \\
& \overline{\bar{y}}(n-P)=\sum_{k=1}^{F} \overline{\bar{a}}_{k} y_{w}(n-P+k)
\end{aligned}
$$

As expressões (6a) e (6b) permitem definir os erros de predição futura, $f_{p}(n)$, e de predição passada, $b_{p}(n)$, de origem $P[12,14]$ : 


$$
\begin{aligned}
& f_{p}(n)=y_{w}(n)-\sum_{k=1}^{P} \bar{a}_{k} y_{w}(n-k) \\
& b_{0}(n)=y_{w}(n-P)-\sum_{k=1}^{P} \overline{\bar{a}}_{k} y_{w}(n-P+k)
\end{aligned}
$$

Os eiros quadráticos médios totais de prediçäo futura de predição passada, de ordem $P, \xi p$ e $\xi_{b}^{p}$ respectivamemte em um intervalo de largura $N$, sāo definicios pelas expressc̄es abaixo:

$$
\begin{aligned}
& \xi_{f}^{p}=\sum_{n=0}^{N-1} p_{p}^{2}(n) \\
& \xi_{b}=\sum_{n=0}^{N-\xi} b_{p}^{2}(n)
\end{aligned}
$$

Aidéia da prediça inear é obter o conjunto os coefieientes $\left\{\bar{z}_{4}\right\}$ ou $\left\{\overline{\bar{a}}_{k}\right\}$ que sirva cono sstmalva dos coeficientes (ak) do sistema V(z), glie modela a seraço do sinal de voz. Comem mencionar que $v(z)$ pode ser representado, adeguadamente, por um modelo composto apenas por pólos [13]:

$$
V(z)=\frac{G}{1-\sum_{k=1}^{p} a_{k} z^{-k}}
$$

hnde 9 e um fator de gamh. A presenca de zercs sm (9) mehroratia a tepreservapa de sons nasals. O denominador da expressá (9) é denominado fitro inverso reiano aos cosficlenes $\left\{a_{4}\right\}$ Existem vários aboritmos propostos para a obtenção das estimatvas $\left\{\bar{a}_{k}\right\}$ ou $\left.\bar{a}_{k}\right\} .0$ citério inilizado 6 de minimizaçäo do erro quadrático medio total. Pode-se mostrar que os coeficientes $\left\{\overline{\bar{a}}_{k}\right\}$ säo iguais aos coeficiente $\left\{\overline{\bar{a}}_{k}\right\}$, nas com a ordem invertida [14]. Por isso, os coeficientes seräo reterenciados por $\left\{\bar{a}_{k}\right\}$ no decorrer deste trabaho e seráo denominados de coeficientes LPC "Linear Prediction Coefficients"). As opçc̄es de cálculo dos coeficientes $\left\{\bar{z}_{k}\right\}$, oferecidas pelo SIR PI, conforme Figura 8, sāo:

88 
Método da Autocorrelaçāo - Algoritmo de Durbin

Métodos Latrice:

Método "Fonwaro" - Minirnizaçāo do Eno de Predição Fưura

Método "Backward" - Minimização do Erro de Predição Passada

Método de lakura

Métode do Mínimo

Método de Burg

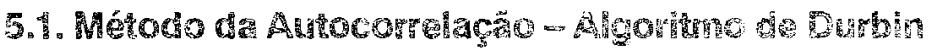

O algorimo baseia-se na minimizaçäo do erro cuadráaico iotal de predicáo

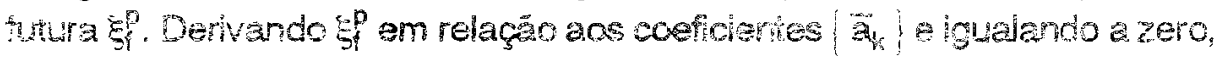
restlta um sistema linear de squacoses, de ordem p.

$$
\sum_{k=1}^{p} a_{k} P(1-k)=n(1) \quad i=1,2, \ldots, p
$$

onde R(i) é o coeficiente de autocorrelagăo de ordemi. O abortmo de Durbin permite !na soluçäo iterativa deste sistema de equefoes [12]:

$$
\xi_{n}^{0}=R(0)
$$

$$
k_{i}=\frac{R(i)-\sum^{-1} a^{(i-1)} F(i-1)}{\xi_{n}^{i-1}}, 4 \leq 1 \leq p
$$

$\bar{a}_{i}^{(i)}=k_{i}$

$$
\bar{a}_{j}^{(i)}=\bar{a}_{i}^{(i-1)}-k_{i} \bar{a}_{i-j}^{(i-1)}, \quad 1 \leq i \leq i-1
$$




$$
\xi_{i n}^{i}=\left(1-k_{i}^{2}\right) \xi_{m}^{i-1}
$$

$$
G=\sqrt{R(0)-\sum_{k=1}^{p} \bar{a}_{k} R(k)}
$$

Os coeficientes $\left\{\bar{u}_{i}^{(i)}\right\}, j=1,2, \ldots, i$; são os coeficientes do preditor de ordem 1. Ri(i) săo os coeficientes de autocorrelação nomalizados em relaçảo à energia do sina! $R(0)$. Os coeficientes $\left\{k_{i}\right\}$ säo denominados coeficientes de refiexāo. O termo $\xi_{\text {fn }}^{i}$ e o erro quadrático médio total de predição futura normalizado em relaçāo a $\mathrm{R}(0)$ e $G$ é fator de ganho da expressāo (9). Uma condição necessária e suficiente para que o sistema $V(z)$ resultante seja estavel é que $\left|k_{i}\right|$ seja menor que 1,0 [11].

\subsection{Métogios Latựce}

Teoricamente, o método da autocorrelaçāo resulta sempre em um sistema $V(z)$ estável. No entanto, o método sotie do efeito do cálculo com precisāo finita, enquanto a formulaça lattice possui uma sensibilidade mulito menor [19]. A formulação látice baseia-se no fato dos erros de prediçăo futura e passada de um preditor de ordem $P$, poderem ser calculados, recursivamente, a partir dos erros de predica futura e passada de preditores de order. menor [1] $19 !$ :

$$
\begin{aligned}
& f_{0}(n)=b_{0}(n)=y(n) \\
& f_{i+1}(n)=f_{i}(n)-k_{i+1} b_{i}(n-1) \\
& b_{i+1}(n)=-k_{i+1} \hat{i}_{i}(n)+b_{i}(n-1)
\end{aligned}
$$

No método lattice a soluçāo é calculada diretamente a partir das amostras do sinal de voz. Náo existe a necessidade de janelamento e portanto, $y_{w}(n)=y(n)$. As derivaçóes possiveis são: 


\subsubsection{Método "Fonward" - Minimização do Erro de Predição Futura}

Este método é a implementação lattice do método da autocorrelaçāo e baseia-se, portanto, na minimizaçăo de $\xi_{\uparrow}^{p}$. Substituindo (12b) em (8a) e derivando em relação aos coeficientes de refiexão $k_{i}$, resulta:

$$
k_{i}^{\dagger}=\frac{\sum_{n=0}^{N-1} f_{i-1}(n) b_{i-1}(n-1)}{\sum_{n=0}^{N-1} b_{i-1}^{2}(n-1)}
$$

\subsubsection{Método "Backward" - Minimização do Erro de Predição \\ Passada}

Neste caso, minimiza-se $\xi_{b}^{p}$. Substituindo (12c) em (8b) e derivando em relaçāo aos coeficientes de reflexāo $k_{i}$, resulta:

$$
k_{i}^{b}=\frac{\sum_{n=0}^{N-1} f_{i-1}(n) b_{i-1}(n-1)}{\sum_{n=0}^{N-1} f_{i-1}(n-1)}
$$

Observa-se que $k_{i}^{q}$ e $k_{i}^{b}$ possuem o mesmo sinal.

\subsubsection{Método de Itakura}

Os coeficientes de reflexāo calculados pelas expressōes (13) e (14) nāo garantem, necessariamente, um filtro estável $V(z)[19]$. Itakura propōe tomar a média geométrica de $k_{i}^{\dagger} \in k_{i}^{b}[14,19]$ :

$$
k_{i}^{\prime}=\operatorname{Sinal}\left(k_{i}^{f}\right) \sqrt{k_{i}^{f} k_{i}^{b}}
$$




\subsubsection{Método do Mínimo}

Neste caso, o mínimo, em valor absoluto, entre $k_{i}^{\dagger}$ e $k_{i}^{b}$ é escolhido:

$$
k_{i}^{M}=\operatorname{Sinal}\left(k_{i}^{f}\right) \text { mínimo }\left\{\left|k_{i}^{\dagger}\right|,\left|k_{i}^{b}\right|\right\}
$$

\subsubsection{Método de Burg}

A soluçāo proposta por Burg minimiza a soma dos erros de predição futura e de predição passada, para cada segmento do sinal de voz e resulta sempre, em sistemas $V(z)$ estáveis [14, 19]:

$$
\xi_{T}^{i}=\xi_{f}^{i}+\xi_{b}^{i}
$$

Substituindo-se (8b) e (8c) em (17a) e derivando-se em relação a $k_{\text {; }}$ resulta [14]:

$$
k_{i}^{8}=\frac{2 k_{i}^{f} k_{i}^{b}}{k_{i}^{f}+k_{i}^{b}}
$$

\subsection{Ordem da Análise LPC}

Aial e Hanauer [13] \& Markel e Gray [14] apresentam uma relaçāo entre a ordem $P$ da análise $L P C$, a freqüêrcia de amostragem $F_{s}$, o comprimento do trato vocálico $T_{v}$ e a velocidade do som no ar $\mathrm{c}$ :

$$
F_{s}=\frac{P c}{2 T_{v}}
$$

O simulador SIR PI permite escolher o valor de P. Na prática são utilizados valores entre 8 e $14[12,13,14]$.

92 

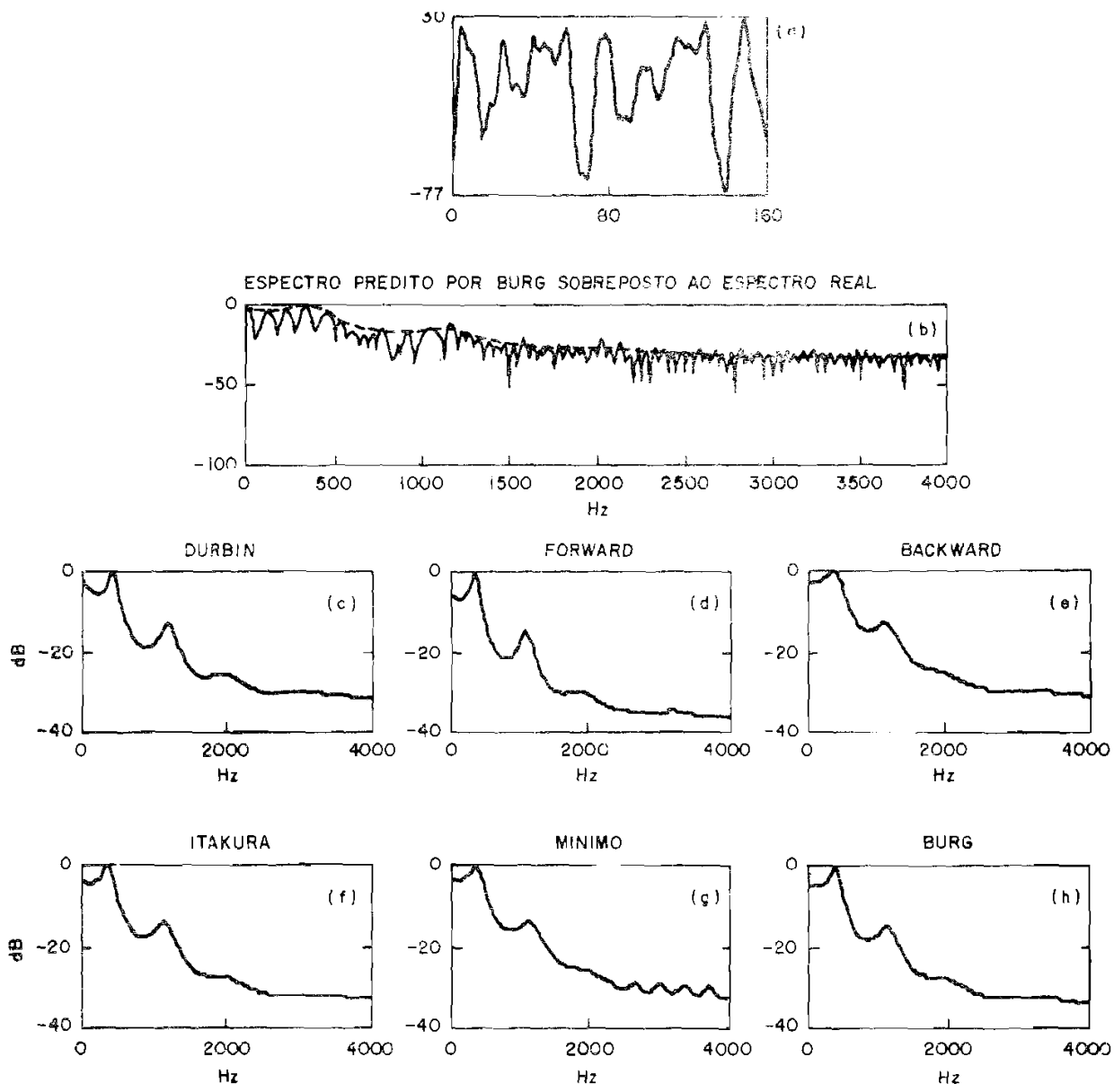

Figura 8. Segmento de 160 amostras (20ms) do som "oi" do dígito "oito" (a), o seu espectro de freqüência (b) e os espectros de $V(z)$ obtidos com os métodos: de autocorrelaçāo (c), "Forward" (d), "Backward" (e), Itakura (f), Mínimo (g) e Burg (h). $P=12$.

\section{MEDIDAS DE SEMELMANÇA}

As medidas de semelhança permitem comparar os parâmetros de dois sinais de vOz com o intuito de verificar se podem ser considerados como representantes da mesma palavra. O simulador SIR PI permite escolher entre várias alternativas. Considerando dois segmentos de voz, um de teste, $t$, e outro de referência, r. tem-se: 


\subsection{Medida Euclideana}

$$
d^{2}=\sum_{k=1}^{P}\left(\vec{a}_{k}^{t}-\vec{a}_{k}\right)^{2}
$$

\subsection{Medida Absoluta}

$$
d_{a}=\sum_{k=1}^{P}\left|a_{k}^{t}-a_{k}^{r}\right|
$$

\subsection{Medida Cepstral}

$$
d_{c}^{2}=\sum_{k=1}^{L}\left(c_{k}^{t}-c_{k}^{r}\right)^{2}
$$

onde Lé o número desejado de coeficientes cepstrais $\left\{c_{k}\right\}$, que podem ser obtidos facilmente a partir dos coeficientes LPC [20].

\subsection{Medida Cepstral Ponderada}

$$
d_{c p}^{2}=\sum_{k=1}^{L} q(k)\left(c_{k}^{t}-c_{k}^{r}\right)^{2}
$$

O simulador SIR PI permite programar a ponderaçāo $q(k)$ e a ordem $L$.

\subsection{Medida da Razão de Verossimilhança}

Esta medida, proposta por Itakura [4], é juntamente com as medidas ceptrais, uma das medidas mais bem sucedidas. A sua idéia é simples e baseia-se na comparaçāo das energias dos erros de prediçāo futura $f_{p}(n)$, nas saídas dos

94 
filtros inversos $A_{Y}(z)$ e $A_{Y}(z)$, de teste e de referência respectivamente, quando o segmento de teste é aplicado a ambos, conforme figura 9 :

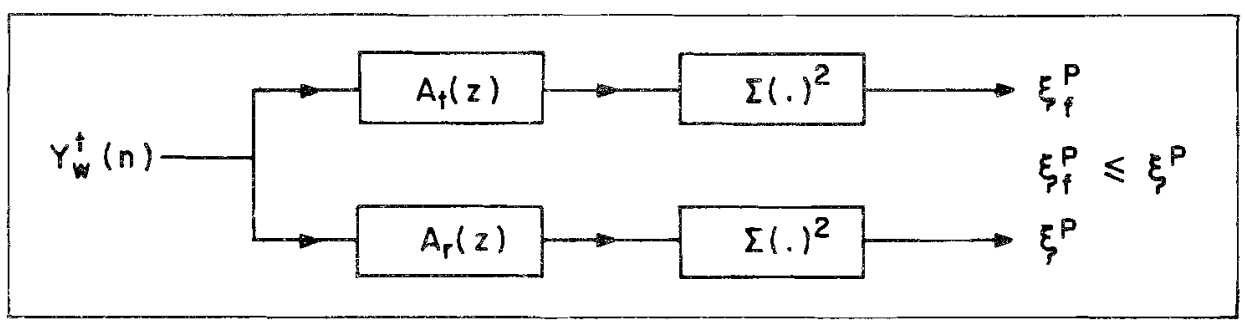

Figura 9. Medida da razāo de verossimilhança de Itakura

A medida de distância é dada pela expressāo abaixo, onde o índice t indica que os erros correspondem à aplicaçāo do segmento de teste nas entradas dos filtros inversos:

$$
\mathrm{d}_{\mathrm{N}}^{t}=\ln \left[\frac{\xi^{p}}{\xi_{p}^{p}}\right]
$$

Percebe-se que a medida proposta por Itakura nāo é uma medida simétrica $\left(d_{N v}^{i} \neq d_{w}^{i}\right)$

\subsection{Medida de Itakura-Saito}

A distância de ltakura-Saito é deñnida pela expressão seguinte:

$$
d_{s}^{\hat{q}}=\left[\frac{G_{i}}{G_{r}}\right]^{2}\left[\frac{\xi_{p}^{p}}{\xi_{f}^{p}}\right]^{i}-2 \ln \left[\frac{G_{t}}{G_{r}}\right]-1
$$

$G_{i}$ e $G_{\gamma}$ são os fatores de ganho dos segmentos de teste e referência, respectivamente (ver expressão (11e)).

Percebe-se, também, que a medida de ltakura-Saito não é simétrica. 


\subsection{Medida $\mathrm{COSH}$}

O objetivo da medica COSH é resolver o problema de assimetria da medida da razāo de verossimthinaça. A medida COSH é definida por:

$$
d_{c o s h}=\ln [1+\Omega+\sqrt[3]{\Omega(2+\Omega)}]
$$

onde $\Omega$ é amedida ds to $d s$.

\subsection{Medida COSBE}

Considerando a razäo entre os fatores de ganho $\left(G_{N} / G_{f}{ }^{2}\right.$ como variável e minimizando 2 em relação a esta variável, resulta [20]:

$$
d_{2} \min =\sqrt{\frac{\left(\xi^{p} / \xi_{p}^{p}\right)^{t}}{\left(\xi^{p} / \xi_{p}^{p}\right)^{r}}}-1
$$

\section{ALINHAMENTO TEMPORAL DINÂMICO}

O principal elemento de um sistema reconhecedor de palavras isoladas é o bloco responsável pela comparação da paavia pronunciada com as palavras do dicionário do sistema (palavra de referência). É fato conhecido que o falante näo consegue, geralmente, repetir a pronúncia de uma mesma palavra, à mesma taxa e com a mesma duração. Esta variação na pronúncia da palavra provoca flutuaçōes nāo lineares ao longo do eixo do tempo [a, 22]. A soluçāo deste problema sugere realizar o alinhamento temporal dinâmico das duas palavras ("Dynamic Time Warping - DW"). $O$ algoritmo geral de alinhamento temporal dinâmico considera duas palavras, una de referência e outra de teste e determina a funçäo ф) (ni) que mapeia a palavra de referência na palavra de teste ou vice-versa. Deve-se escoher a hunçăo que realiza o mapeamento com a menor distância total $D_{T}$, entre as duas palavras $[2 i, 22$, 23]. Em termos gerais, a distância hotale a soma das distâncias iocais ao longo da trajetória.

Para tal, a cada ponto (i, i), do plano de mapeamento, atribui-se uma soma parcial $S_{i}$, que mede a distância acumulada até o ponto $(i, j)$ : 


$$
\begin{aligned}
& S_{i}=d_{i}+\text { minimo }\left\{S_{p q}\right\} \text {, para } p \leq i \text { e } q \leq j \\
& S_{11}=d_{11} \\
& D_{T}=\text { mínimo }\left\{S_{p q}\right\}, \text { para } p=\text { última coluna }
\end{aligned}
$$

onde $d_{i j}$ representa a distância local entre os segmentos $i$ (eixo horizontal) $e$ j (eixo vertical) do plano (i, i) e $S_{p q}$ representa a distância acumulada nos pontes $(p, q)$ à esquerda e abaixo de $(i, j)$. Para garantir que näo ocorram expansōes ou compressōes excessivas, a inclinação de ф(n) deve situar-se, preferencialmente, na faixa $\left[\frac{1}{2}, 2\right]$ [22]. Restriçōes locais sāo ainda, impostas para especificar quais sāo os pontos $(p, q)$ que devem ser considerados na minimizaçāo de $S_{p q}$. Existem vários tipos de restriçóes locais, admitidas pelo SIR PI, conforme figura $10[4,22,23]$.

Outra restriçāo geralmente imposta, é o alinhamento dos segmentos extremos das duas palavras. Supondo, sem perda de generalidade, que os segmentos das palavras dos eixos horizontal e vertical estāo indexados de 1 a $N_{h}$ e de 1 a $N_{v}$, respectivamente, entáo o alinhamento dos extremos implica nas seguintes restriçōes [21]:

$$
\begin{aligned}
& 1 \leq \Phi(1) \leq \delta+1 \\
& N_{v}-\delta \leq \Phi\left(N_{h}\right) \leq N_{v}
\end{aligned}
$$

As condiçōes (29), em conjunto com a restriçāo de inclinaçāo da funçāo de mapeamento, restringem a área de procura da trajetória ótima à regiāo hachurada da figura 11 . As duas retas parajelas que reduzem a área de procura são uma imposição adicional para diminuir o tempo de processamento necessário.

O fator $\theta$, denominado pelo SIR PI de fator global, representa a máxima diferença absoluta permiticia entre os segmentos de teste e de referência.

$$
|i-j| \leq \theta
$$




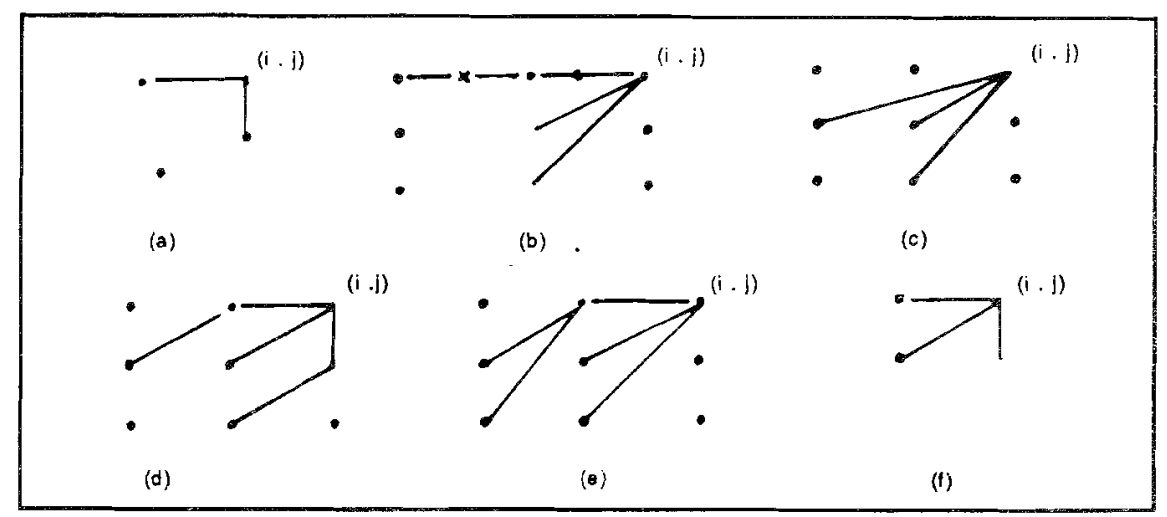

Figura 10. Restriçōes locais admitidas pelo simulador. Horizontal-Vertical (HV) (a), Itakura (b) 3 Diagonais (c), Horizontal-Vertical-3 Diagonais (HV3D) (d), Horizontal-4 Diagonais (H4D) (e) e Horizontal-Diagonal-Vertical (HV1D) (f).

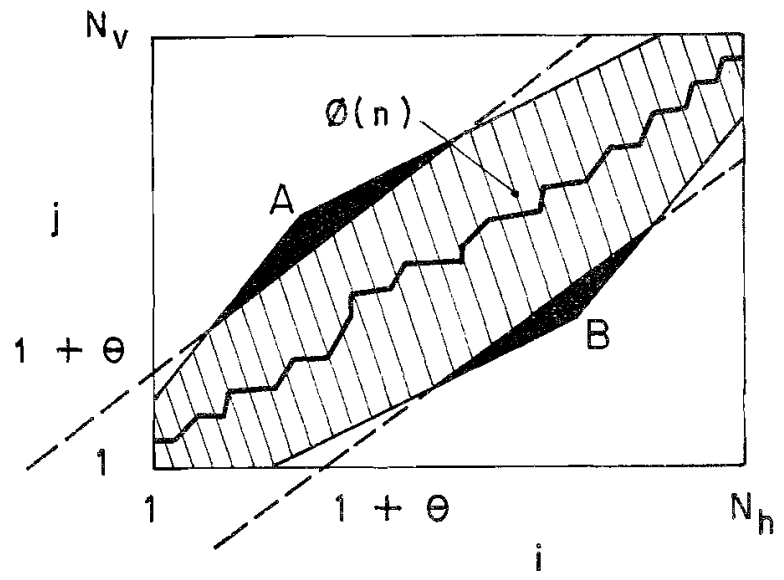

Figura 11. Regiāo de procura da funçāo $\phi$ (n)

Myers e Rabiner [23] mostram que a reduçāo da área de procura toma-se vantajosa se os comprimentos das duas palavras forem normalizados para um mesmo valor, antes da aplicaçáo do algorítmo de alinhamento temporal dinâmico. Esta normalizaçāo pode ser feita por interpolaçāo ou por dizimação. Outro ponto importante a ser mencionado, refere-se à opçāo de se alinhar a palavra de teste ao longo do eixo horizontal ou ao longo do eixo 
vertical. Sabe-se que a escolha influi no resultado final. Existe, então, a necessidade de se normalizar a distância total com relaçäo ao número de segmentos da palavra que está ao longo do eixo horizontal, para que a distância total represente uma distância média ao longo da trajetória [22, 23]. Esta normalizaçāo depende do tipo da funçāo de ponderação das condiçōes locais, que se está empregando. Em outras palavras, pode-se auribuir pesos aos ramos de transiçăo das condiçōes locais da Figura 10. Existem várias funçōes de ponderaçāo. O peso de um ramo $r, \Lambda^{r}$, pode ser dado por [22, 23]:

$$
\begin{aligned}
& \text { Mínimo }(\Delta x, \Delta y): \Lambda^{r}=\text { mínimo }\left(\Delta x^{r}, \Delta y^{r}\right) \\
& \text { Máximo }(\Delta x, \Delta y): \Lambda^{r}=\text { máximo }\left(\Delta x^{r}, \Delta y^{r}\right) \\
& \Delta x: \Lambda^{r}=\Delta x \\
& \Delta x+\Delta y: \Lambda^{r}=\Delta x^{r}+\Delta y^{r}
\end{aligned}
$$

Os termos $\Delta x^{\prime} \in \Delta y^{\prime}$ representam a variaçāo das coordenadas $x$ e y ao longo do ramo $r$. Costuma-se adotar os seguintes valores para o fator de rormali.zaçāo, NR, em funçāo da funçāo de ponderaçāo:

$$
\begin{aligned}
& \text { Mínimo }(\Delta x, \Delta y): N R=N_{q} \\
& \text { Máximo }(\Delta x, \Delta y): N R=N_{q} \\
& \Delta x: N R=N_{q} \\
& \Delta x+\Delta y: N R=N_{q}+N_{v}
\end{aligned}
$$

onde $N_{q}$ é o ponto onde a funçáo de mapaamento aünge o valor máximo $N_{v}\left(N_{q} \leq N_{h}\right)[21]$, is:o é:

$$
\phi\left(N_{Q}\right)=N_{v}
$$

Com isso, a expressāo geral da distância total toma-se:

$$
D_{T}=\frac{\sum d_{i} \Lambda^{r}}{\operatorname{NA}(\Lambda)}, \text { para }(i, j) \text { e } \phi(n)
$$


O simulador SIR PI permite controlar o bloco de alinhamento temporal dinâmico e programar todos os parâmetros de interesse.

\section{REGRAS DE DECISÃO}

O último passo no sistema de reconhecimento de palavras isoladas é o processo de decisão. Este passo encarrega-se de analisar as distâncias totais, calculadas pelo bloco de DTN, entre a palavra de teste e as palavras de referencia, e decidir qual a palaura do dicionário que mais se aproxima do comando falado. As regras de decisäo mas populares são a regra NN ("Nearest Neighbour") e a regra KNN ("K Nearest Neighbour") [1, 8, 24]. As duas regras estáo implementadas no SIR PI.

\section{VISÄO GLOBAL DO RECONHECEDOR}

A Figura 12 apresenta o diagrama em biocos do sistema reconhecedor de palavras isoladas. Todos os blocos forani discutidos nos itens anteriores.

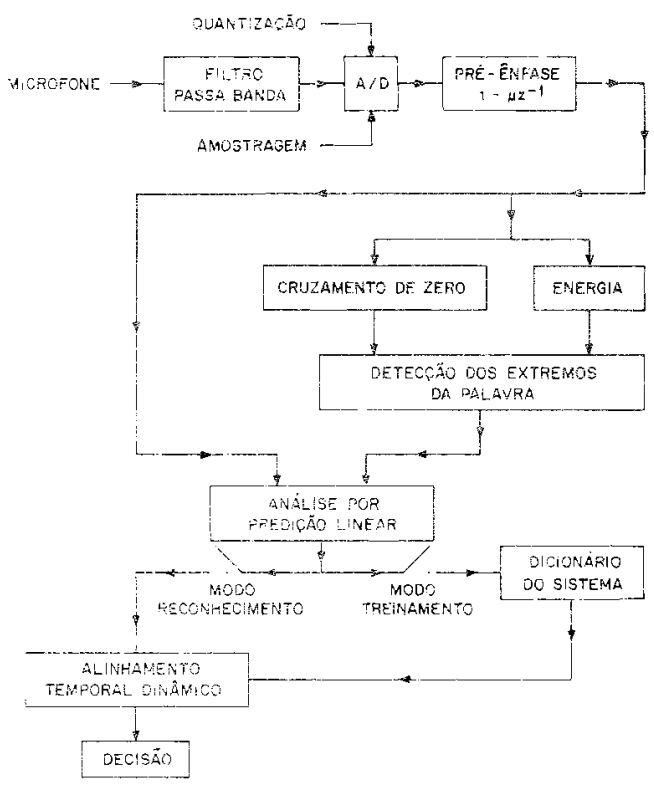

Figura 12. Diagrama am blocos do reconhecedor 
Deve-se mencionar, neste ponto, que o reconhecedor possui dois modos de operaçäo: o modo de treinamento e o modo de reconhecimento. No modo de treinamento, as palavras de referência são pronunciadas e analisadas e os seus coeficientes LPC sáo gerados a armazenados. No modo de reconhecimento, os coeficientes LPC da palavra pronunciada são gerados e o processo de análise continua até o passo de decisão.

\section{TESTE DO SIMULADOR}

Neste item, sāo apresentados os resultados obtidos durante a simulaçáo de uma configuraçẫo îreqüente na literatura. Os parâmetros da configuraçāo testada estáo listados a seguir:

LPC: Método de Durbin, janela de Hamming com $N=160,0=80$ e $p=8$

DTW: Distância cepstral com $L=8$, restrição de itakura com ponderaçäo $\Delta x$, regra NN ou KNN de decisẵo, palava de teste ao longo do eixo horizontal, limitação global desativada, pontos extremos vinculados $(\delta=0)$, e normalizaçäo de comprimentos ativada

"Energia": Medida absoluta, janela retangular $\operatorname{com} N=80$ e $D=30$, e fator de pré-ènáse $\mu=0,95$.

O sinal de voz é fitrado entre 300 e $3400 \mathrm{~Hz}$, mostrado a $8000 \mathrm{~Hz}$ quantizado em $12 \mathrm{bits}$. As palavras de teste ce refrêncla foram levantadas com o auxilio de 8 fajantes designados $A, B, C, \times 1, \times 2, \times 3, \times 4,35, O s$ talantes $A, B, \times 1, \times 2$ e $X 3$ são masculinos, enquento os palantes $6, X 4$ e $\times 5$ săo femininos. As palavras utilizadas nos testes ato as digitos de ZERO a NOVE as palavras MEIA, AJUDA, CANCELE T TEFMINE Cada falante do grupo $(A, B, C$ ) pronunciou cade palavra 6 vezes. Portanto, a cada falante $A, B$ ou $C$ correspondern 6 conjuntos da 14 pronuncias, designadas $A, B_{i}, e C_{i}, i=1,2, \ldots, 6$.

Cada falanie do grupo $\left.\{X\}, \times 2, X_{3}, X_{4}, X 5\right\}$ pronuncion cada palavra una vez. Os testes foram realizadce em um microcompltador compativel com o PC da $1 \mathrm{BM}^{1}$ (8 MHz, microprocessador $286 \mathrm{da} \mathrm{intel}^{2}$ e com coprocessador). O sempo

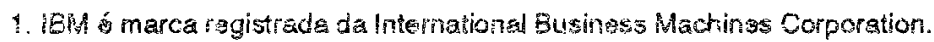

2. intel á marca registrada da Irtel Corporation.

Revisla da Scciedade Brasibaira de Tebcomuniceçoes

Volums $6, N^{0} 1$, dezembro de 1991 
médio gasto na análise de uma palavra de teste (pré-ênfase, detecção dos pontos extremos e cálculo dos parâmetros LPC) foi de 20 segundos. Ó tempo médio gasto para alinhar dinamicamente duas palavras fol de 22 segundos. Em média, foram necessários $6 \mathrm{Kbytes}$ de memória, em disco, por palavra de referência. A seguir são apresentados os resultados obtidos:

Dicionários I.A, I.B e I.C:

Os coniuntos $A_{6}, B_{6}, C_{6}$ foram escolhidos como referência para gerar três dicionários I.A, I.B e I.C. respectivamente. Os 5 conjuntos restantes, de cada falante, foram utilizados para testar o dicionário correspondente. Os resultados estão listados na tabela 1:

\begin{tabular}{|c|c|c|}
\hline Dicionário & $\begin{array}{c}\text { Número de palavras não } \\
\text { ieconhecidas }\end{array}$ & Taxa de acerio \\
\hline I.A & 0 & $100 \%$ \\
B & 4 & $94 \%$ \\
\hline
\end{tabular}

Tabela 1. Testes dos dicionários |

Portanto a taxa média de acerto é da ordem de $96 \%$.

Dicionário !l:

Os coriuntos $A_{6}, B_{6} \in C_{6}$ foram escolhidos como referência para gerar o dicionário ll. Os 5 coniuntos restantes, de cada falante, foram utilizados para testar o dicionário. (210 palavres de teste). Apenas quatro palavras de teste não foram reconhecidas corretamente, o que indica uma taxa de acerto de $98 \%$. Percebe-se que a laxa de acento aumentou de $96 \%$ para $98 \%$ devido ao número majo de referências por comando.

\section{Dicionário lll:}

Os conjuntos $A_{6}, B_{6}, C_{6}, A_{4}, B_{4}$ a $C_{4}$ foram escolhidos como reterência para gerar o dicionário 111 . As pronúncias dos falantes $\times 1$ a $\times 5$ foram utilizadas nos 
testes do dicionário (70 palavras de teste, no total). Foram realizados dois testes: um teste com a regra NN e outro teste com a regra KNN (K=3). Para ambos os casos apenas três palavras de teste nāo foram reconhecidas corretamente, o que indica uma taxa de acerto da crdem de $95 \%$.

Os testes realizados mostram que a configuração testada fornece bons índices de reconhecimento e confirmam os resultados obtidos por vários pesquisadores $[4,5,6,7,9,10]$.

Convém ressaltar que os resultados apresentados neste item referem-se ao teste de uma configuraçăo. Outras configuraçōes podem também ser programadas e testadas.

\section{INTERFACE DO SIMULADOR COM O USUÁRIO}

A operaçáo do simulador é bastante simples e a sua programação é iotalmente controlada por janelas ou menus. O simulador [1] fol desenvolvido em um microcomputador compatível com o PC da IBM' e escrito em linguagem C (Mierosoft ${ }^{2}$ versảo 5.10 ). 0 simulador possui aproximadamente 10.000 linhas de código.

\section{CONCLUSÖES E COMENTÁRIOS}

Neste trabalho foi discutido o desenvolvimento de um simulador de reconhecedores de comandos da fala. Os parâmetros que controlam a operaçāo do sistema sāo facilmente programados pelo usuário e a configuraçāo escolhida pode ser facilmente simulada e avaliada. A nova versáo do simulador se encontra em desenvolvimento e deverá incluir a geração de dicionários independentes de falantes $[6,8,25,26,27]$.

O simulador desenvolvido [1] permite testar muitos dos algoritmos apresentados na literałura e representa, portanto, uma contribuição na área de reconhecimento de voz.

1. IBM é marca registrada da International Business Machines

2. Microsoft é marca registrada da Microsoft Corporation 


\section{AGRADECIMENTOS}

Ao Prof. Dr. Zsolt L. Kovacs pelo incentivo e discussōes, ao Prof. Edgard José Casaes pela colaboração na área de linguística, ao Eng. Ivandro Sanches pela troca de idéias e aos engenheirandos Wander $O$. Cesário, Alex $D$. Zyrianoff e Roberto Martinelli pela colaboraçăo.

\section{REFERÊNCLAS BIBLIOGRÁFICAS}

[1] A.H. Sayed, "Simulador de Reconhecedores de Palavras Isoladas - Sir PI," Dissertação de Mestrado. Departamento de Engenharia de Eletricidade da EPUSP, Julho 1989.

[2] D.R. Reddy, "Speech recognition by Machine: A Review" Proceedings of the IEEE, vol. 64, pp. 501-531, April 1976.

[3] G.M.White, "Speech Recognition: A Tutorial Ovenview", Computer, vol. 9, pp. 40-53, May 1976.

[4] F. Itakura, "Minimum Prediction Riesidual Principle Applied to Speech Recognition", IEEE Transactions on Acoustics, Speech and Signal Processing, vol. ASSP-23, n 1, pp. 67-72, February 1975.

[5] M.R. Sambur and L. R. Rabiner, "A Speaker Independent Digit Recognition System", The Bell System Technical Journal, vol. 54, n 1, pp. 91-103, January 1976.

[6] L.A. Rabiner, "On creating Reference Templates for Speaker Independent Recognition of !solated Words", IEEE Transactions on Acoustics, Speech and Signal Processing, vol. ASSP-26, n1, pp. 34-41, February 1978.

[7] L.R. Fabiner, J.G. Wilpon and A.E. Fosenberg, "A Voice Controlled Repertory Dialer System", The Bell System Technical Joumal, vol. 59, pp. 1153-1163, September 1980 .

[8] L.R. Fabiner and S.E. Levinson, "Isclated and Connected Word Recognition - Theory and Selected Applications", IEEE Transactions on Communications, vol. COM-29, n 8, pp. 621-658, May 1981.

[9] Y.Tohkura, "A Weighted Cepstral Distance Measure for Speech Fecognition", IEEE Transactions on Acoustics, Speech and Signal Processing, vol. ASSP-35, n 10, pp.1414-1422, October 1987. 
[10] J. G. Wilpon, L.R. Rabiner and A. Bergh, "Speaker Independent Isolated Word Reccgnition Using a 129-Word Airline Vocabulary", The Jorunal of the Acoustical Society of America, vol. 72, n2, pp. 390-396, Augusi 1982.

[11] J.L. Flanagan, Speech Analysis, Synthesis and Perception, Springer-Verlag, New York, 1972.

[12] L.R. Rabiner and R.W. Schafer, Digital Processing of Speech Signals, Prentice Hall: New Jersey, 1978.

[13] B.S. Atal and S.L. Hanauer, "Speech Analysis and Synthesis by Linear Prediction of the Speech Wave", The Joumal of the Acoustical Saciety of America, vol, 50, n 2, part 2, pp. 637-655, April 1971.

[14] J.D.Markel and A.H. Gray, Jr. Linear Prediction of Speech, Springer Verlag, New York, 1976.

[15] R.W. Schafer and L.R. Rabiner, "Digital Representations of Speech Signals", Proceedings of the IEEE, vol. 63, pp. 662-677, April 1975.

[16] A.V. Oppenheim and R.W. Schafer, Digital Signal Processing, PronticeHaill, New Jersey, 1975.

[17] A.H. Sayed A. Ferrara e E.T. Taniguchi, "Sisterna Reconhecedor de Voz Humana - Programável e com Capacidade de Processannento", Projeto de Formatura, Departamento de Engenharia de Eletricidade da EPUSP, 1987.

[18] L.R. Rabiner and M. R. Sambur, "An Algorithm for Determining the End oints of I solated Utterances", The Bell System Technical Journal, vol. 54, n 2, pp. 297-315, February 1975.

[19] J. Makhoul, "Stable and Efficient Lattice Methods ior Linear Prediction", IEEE Transactions on Acoustics, Speech, and Signal Processing, vol. ASSP-25, n 5, pp. 423-428, October 1977.

[20] A.H. Gray, Jr. and J.D. Markel, "Distance Measures for Speech Processing", IEEE Transactions on Acoustics, Speech, and Signal Processing, vol. ASSP-24, n 5, pp. 380-391, October 1976.

[21] L. Rabiner, A.E. Rosenberg and S.E. Levinson, "Considerations in Dynamic Time Algorithms for Discrete Word Recognition", IEEE Transactions on Acoustics, Speech and Signal Processing, vol. ASSP.26, n6, pp. 676-592, December 1978. 
[22] H. Sakoe and S. Chiba, "Dynamic Programming Algorithm Optimization for Spoken Word Recognition", IEEE Transactions on Acoustics, Speech and Signal Processing, vol. ASSP-26, pp. 43-49, February 1978.

[23] C. Myers, L.R. Rabiner and A.E. Rosenberg, "Performance Tradeoffs in Dynamic Time Warping Algorithms for Isolated Word Recognition", IEEE Transactions on Acoustics, Speech and Signal Processing, vol. ASSP-29, n 6, pp. 623-635, December 1980.

[24] V.N. Gupta, J.K Bryan and J.N. Gowdy, "A Speaker-Independent Speech Recognition System Based on Linear Prediction". IEEE Transactions on Acoustics, Speech and Signal Frocessing, vol. ASSP-26, n 1, pp. 27-33, February 1978.

[25] B.H. Juang, D.Y. Wong and A.H. Gray, Jr., "Distortion Performance of Vector Quartization for LPC Voice Coding", IEEE Transactions on Acoustics, Speech and Signal Processing, vol. ASSP-30, n 2, pp. 294-303, April 1982.

[26] Y. Linde, A. Buzo and R. M. Gray, "An Algorithm for Vector Quantizer Design", IEEE Transactions on Communications, vol. COM-28, $\mathrm{n} 1, \mathrm{pp}$. 84-95, January 1980.

[27] A. Buzo, A.H. Gray, Jr., R.M. Gray and J.D. Markel, "Speech Coding Based Upon Vector Quantization", IEEE Transactions on Acoustics, Speech and Signal Processing, vol. ASSP-28, n 5, pp. 562-573, October 1980. 